Check for updates

Cite this: J. Mater. Chem. A, 2018, 6 , 5703

Received 9th January 2018

Accepted 4th March 2018

DOI: $10.1039 / \mathrm{c} 8 \mathrm{ta0} 0265 \mathrm{~g}$

rsc.li/materials-a

\title{
A full monolayer of superoxide: oxygen activation on the unmodified $\mathrm{Ca}_{3} \mathrm{Ru}_{2} \mathrm{O}_{7}(001)$ surface $\uparrow$
}

\author{
Daniel Halwidl, (D) a Wernfried Mayr-Schmölzer, ${ }^{\text {ab }}$ Martin Setvin, ${ }^{a}$ David Fobes, (D) \\ Jin Peng, ${ }^{c}$ Zhiqiang Mao, ${ }^{c}$ Michael Schmid, ${ }^{a}$ Florian Mittendorfer, (DD ab \\ Josef Redinger $^{\mathrm{ab}}$ and Ulrike Diebold (D) *a
}

\begin{abstract}
Activating the $\mathrm{O}_{2}$ molecule is at the heart of a variety of technological applications, most prominently in energy conversion schemes including solid oxide fuel cells, electrolysis, and catalysis. Perovskite oxides, both traditionally-used and novel formulations, are the prime candidates in established and emerging energy devices. This work shows that the as-cleaved and unmodified $\mathrm{CaO}$-terminated (001) surface of $\mathrm{Ca}_{3} \mathrm{Ru}_{2} \mathrm{O}_{7}$, a Ruddlesden-Popper perovskite, supports a full monolayer of superoxide ions, $\mathrm{O}_{2}{ }^{-}$, when exposed to molecular $\mathrm{O}_{2}$. The electrons for activating the molecule are transferred from the subsurface $\mathrm{RuO}_{2}$ layer. Theoretical calculations using both, density functional theory (DFT) and more accurate methods (RPA), predict the adsorption of $\mathrm{O}_{2}{ }^{-}$with $E_{\text {ads }}=0.72 \mathrm{eV}$ and provide a thorough analysis of the charge transfer. Non-contact atomic force microscopy (nc-AFM) and scanning tunnelling microscopy (STM) are used to resolve single molecules and confirm the predicted adsorption structures. Local contact potential difference (LCPD) and X-ray photoelectron spectroscopy (XPS) measurements on the full monolayer of $\mathrm{O}_{2}{ }^{-}$confirm the negative charge state of the molecules. The present study reports the rare case of an oxide surface without dopants, defects, or low-coordinated sites readily activating molecular $\mathrm{O}_{2}$.
\end{abstract}

\section{Introduction}

Oxygen plays a pivotal role in solid oxide fuel cells, electrolysis and catalysis, where complex ternary oxides are intensively investigated materials. ${ }^{1}$ Of particular interest are perovskitetype oxides with the basic formula $\mathrm{ABO}_{3}$ (where $\mathrm{A}$ stands for an alkali, alkaline earth, or rare earth metal, while B refers to a transition metal) and variations of this structure, such as double perovskites, $\mathrm{A}^{\prime} \mathrm{A}^{\prime \prime} \mathrm{B}_{2} \mathrm{O}_{5+\delta}$ or the Ruddlesden-Popper series $\mathrm{A}_{n+1} \mathrm{~B}_{n} \mathrm{O}_{3 n+1} .{ }^{1}$ Molecular $\mathrm{O}_{2}$ in its triplet ground state is an inert species and requires activation to undergo catalytic reactions or dissociation into atomic oxygen. ${ }^{2}$ The $\mathrm{O}_{2}$ activation is a crucial step in the oxygen reduction reaction ${ }^{3}$ (ORR) that is potentially a major rate limiting factor for applications. ${ }^{4}$ Therefore a microscopic understanding of the oxygen surface chemistry on perovskite-type oxides is of great importance. ${ }^{5}$

In general, the activation of $\mathrm{O}_{2}$ is achieved by either exciting the neutral molecule to one of its two singlet states ${ }^{6}$ or by charging the molecule thus forming a superoxide $\left(\mathrm{O}_{2}{ }^{-}\right)$or

\footnotetext{
${ }^{a}$ Institute of Applied Physics, TU Wien, Wiedner Hauptstrasse 8-10/134, 1040 Vienna, Austria.E-mail: diebold@iap.tuwien.ac.at

${ }^{b}$ Center for Computational Materials Science, TU Wien, Wiedner Hauptstrasse 8-10/ 134, 1040 Vienna, Austria

${ }^{c}$ Department of Physics and Engineering Physics, Tulane University, 2001 Percival Stern Hall, New Orleans, LA 70118, USA

$\dagger$ Electronic supplementary information (ESI) available: An STM movie in avi format is available (Movie S1). See DOI: 10.1039/c8ta00265g
}

a peroxide $\left(\mathrm{O}_{2}{ }^{2-}\right)$ ion. If available, the electrons for charging the $\mathrm{O}_{2}$ can be transferred directly from the oxide surface, but a substantially unperturbed oxide (i.e. stoichiometric, step- and defect-free) rarely provides electrons for a spontaneous formation of charged oxygen species. Often the oxide has to be activated by photoinduced electron transfer, surface intermolecular electron transfer, ${ }^{7}$ or the introduction of dopants, defects, or low-coordinated sites. ${ }^{8}$

Superoxo and peroxo species have been predicted and/or observed on a few catalytically relevant binary oxides. In experiments on anatase $\mathrm{TiO}_{2}(101)$ it was recently experimentally shown that molecular oxygen accepts charge from subsurface $\mathrm{Nb}^{+}$dopants, resulting in a mixed adsorption of neutral $\mathrm{O}_{2}$ and superoxide ions ${ }^{9}$ at low temperature $(5 \mathrm{~K})$. Charged oxygen species have been predicted for reduced $\mathrm{CeO}_{2}(111),{ }^{10}(110)$ and (100) surfaces, ${ }^{11}$ and their formation has been experimentally confirmed on polycrystalline $\mathrm{CeO}_{2} \cdot{ }^{12,13}$ Calculations for pure $\mathrm{ZrO}_{2}$ show only a weak physisorption of $\mathrm{O}_{2}$ while yttria stabilized zirconia should facilitate dissociation via a superoxo species. ${ }^{14}$ Similarly, $\mathrm{O}_{2}$ is predicted to adsorb weakly on pure $\mathrm{ZnO}(10 \overline{1} 0)^{15}$ but to form a superoxo species when the $\mathrm{ZnO}$ is Aldoped. ${ }^{16}$ Charged oxygen species on $\mathrm{SnO}_{2}$ play a crucial role in chemical sensing, although the correct interpretation of experimental results has been questioned. ${ }^{17}$

The binary alkaline-earth oxide (001) surfaces are closely related to AO-terminated perovskite surfaces. Molecular oxygen does not adsorb on the unperturbed $\mathrm{CaO}(001)^{18}$ and $\mathrm{MgO}(001)^{19}$ 
surface. Only after introducing defects by ultraviolet or gamma irradiation, thermal activation, or doping with transition metal ions the formation of charged oxygen species was observed. ${ }^{\mathbf{1 8 , 2 0}}$ Ultrathin $\mathrm{MgO}$ films supported on a metal (Ag or Mo) allow for an electron transfer from the substrate to activate adsorbed oxygen, explaining the catalytic properties of such systems. ${ }^{19,21,22}$

Turning to ternary oxides, direct experimental evidence on microscopic reaction pathways is sparse. One of the most studied perovskite oxides is $\mathrm{SrTiO}_{3} .{ }^{5}$ Calculations have shown that on the technologically relevant SrO-terminated (001) surface $\mathrm{O}_{2}$ is activated to $\mathrm{O}_{2}{ }^{-}$only if surface oxygen vacancies are present. $^{23}$ In contrast, the defect free LaO-terminated $\mathrm{La}_{2} \mathrm{NiO}_{4}(001)$ surface is theoretically predicted to readily chemisorb oxygen either as superoxo or peroxo species. ${ }^{24}$

This paper combines theory and experiment to investigate on the first step in oxygen activation on a ternary oxide, namely electron transfer to adsorbed $\mathrm{O}_{2} \cdot \mathrm{Ca}_{3} \mathrm{Ru}_{2} \mathrm{O}_{7}$, the prototypical perovskite material considered here, is a Ruddlesden-Popper perovskite (alternating $\mathrm{ABO}_{3}$ and $\mathrm{AO}$ layers) and cleaves easily between adjacent $\mathrm{CaO}$ layers. It is shown that the pristine $\mathrm{CaO}$ terminated (001) surface facilitates the activation of $\mathrm{O}_{2}$ to a superoxide ion without a need for prior surface treatment, or the presence of steps, oxygen-vacancies or dopants. Surface spectroscopy in combination with atomically-resolved Scanning Probe Microscopy and DFT calculations confirm the charge state of the as-formed oxygen and provide detailed models for the adsorption geometry of isolated molecules and the dense $(2 \times 1) \mathrm{O}_{2}{ }^{-}$overlayer formed at higher coverage.

\section{Methods}

\section{DFT calculations}

The surface was modeled using one double-layer thick slabs of $\mathrm{Ca}_{3} \mathrm{Ru}_{2} \mathrm{O}_{7}$, separated by $15 \AA$ of vacuum. The calculations were performed with the Vienna $A b$ initio Simulation Package ${ }^{25}$ (VASP). The van-der-Waals contributions were described by the optB86 $b^{26,27}$ functional which has been employed successfully in previous studies of water adsorption on ruthenate surfaces. ${ }^{28,29}$ The low-coverage case was described using a $(3 \times$ 3) unit cell with one $\mathrm{O}_{2}$ molecule adsorbed on it, yielding a coverage of $1 / 9$, while the high-coverage model cell consisted of a $(2 \times 1)$ surface unit cell with two added $\mathrm{O}_{2}$ molecules. For the large $(3 \times 3)$ unit cell a $2 \times 2 \times 1$ Gamma-centered $k$-point grid was sufficient to achieve electronic convergence while for the smaller $(2 \times 1)$ model a $3 \times 6 \times 1$ Gamma centered mesh was used. The energy cutoff was set to $400 \mathrm{eV}$. The hybrid calculations were performed using the range-separated HSE06 (ref. 30) functional which implements the Fock exchange interaction in the PAW formalism. ${ }^{25}$ For the advanced manyelectron calculations a modern, low-scaling algorithm was used to obtain both the ACFDT-RPA total energies ${ }^{31,32}$ and the $\mathrm{G}_{0} \mathrm{~W}_{0}$ densities of states. ${ }^{33}$ Due to the computational cost involved, the $k$-point grid was reduced to a $2 \times 4 \times 1$ mesh for the $(2 \times 1)$ model cell for both the hybrid functional (HSE06) and the many-electron $\left(\mathrm{G}_{0} \mathrm{~W}_{0} / \mathrm{RPA}\right)$ calculations. The $\mathrm{O} 1 \mathrm{~s}$ core level binding energies were calculated in the initial state approximation.

\section{Experimental setup}

XPS and STM measurements were carried out in an ultrahigh vacuum (UHV) system consisting of a preparation chamber and an STM chamber with base pressures of $2 \times 10^{-11}$ and $6 \times$ $10^{-12}$ mbar, respectively. A low-temperature STM (commercial Omicron LT-STM) was operated at $78 \mathrm{~K}$ in constant-current mode using an electrochemically etched W-tip. XPS was performed in the preparation chamber using nonmonochromatized $\mathrm{Mg} \mathrm{K} \alpha$ X-rays and a SPECS PHOIBOS 100 electron analyser at normal emission with a pass energy of $15 \mathrm{eV} . \mathrm{O}_{2}$ was dosed in the preparation chamber while keeping the sample at $110 \mathrm{~K}$.

Combined STM/nc-AFM measurements were performed at $4.8 \mathrm{~K}$ in a UHV system consisting of a preparation chamber and an analysis chamber with base pressures below $2 \times 10^{-11} \mathrm{mbar}$, equipped with a commercial Omicron q-Plus LT head. Tuningfork-based AFM sensors with a separate wire for the tunnelling current were used $^{34}\left(k=1900 \mathrm{~N} \mathrm{~m}^{-1}, f_{0}=31500 \mathrm{~Hz}, Q \approx\right.$ 30 000). A custom-design cryogenic differential preamplifier was used for measuring the cantilever deflection. ${ }^{35}$ Electrochemically etched W-tips were glued to the tuning fork and cleaned in situ by field emission and self-sputtering in $10^{-6}$ mbar $\mathrm{Ar}^{36} \mathrm{O}_{2}$ was either dosed directly into the cryostat in the analysis chamber at $5.5 \mathrm{~K}$ or in the preparation chamber while keeping the sample at $123 \mathrm{~K}$.

For all STM measurements the bias voltage was applied to the sample; positive or negative bias voltages result in STM images of the unoccupied or occupied states, respectively. All STM images shown were corrected for distortions as described elsewhere. ${ }^{37}$

\section{Sample preparation}

High-quality calcium ruthenate single crystals were grown by the floating zone technique using a mirror-focused furnace. ${ }^{38}$ The composition of the samples was determined via inductively coupled plasma mass spectroscopy (ICP-MS) using laser ablation (LA) for direct analysis of the solid samples. Before insertion into the UHV the samples were fixed on stainless-steel sample plates with conducting silver epoxy glue (EPO-TEK H21D, Epoxy Technology Inc.), and a metal stud was glued on top with another epoxy adhesive (EPO-TEK H77, Epoxy Technology Inc.). The crystals were cleaved in UHV at $110 \mathrm{~K}$ by removing the metal stub with a wobble stick.

\section{Results}

\section{The pristine surface}

As a sample, $\mathrm{Ca}_{3} \mathrm{Ru}_{2} \mathrm{O}_{7}$ was chosen. This is the $n=2$ member of the Ruddlesden-Popper series $\mathrm{Ca}_{n+1} \mathrm{Ru}_{n} \mathrm{O}_{3 n+1}$, which consists of $n$ perovskite-like $\mathrm{CaRuO}_{3}$ layers separated by adjacent $\mathrm{CaO}$ layers along the [001] direction (orthorhombic unit cell: $a=$ $5.365 \AA, b=5.562 \AA, c=19.525 \AA$ ). The $\mathrm{RuO}_{6}$ octahedra are alternately tilted with respect to the $c$ axis, and alternately rotated in the $a b$ plane, ${ }^{39}$ where the $a, b$ and $c$ axes correspond to the [100], [010] and [001] directions, respectively, see Fig. 1a, b. The preferred cleaving plane is between two $\mathrm{CaO}$ layers, with 
a

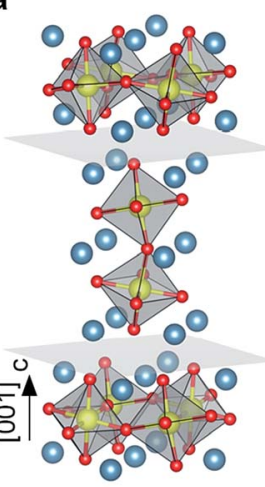

c

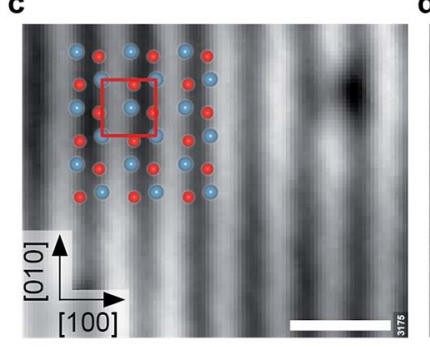

b

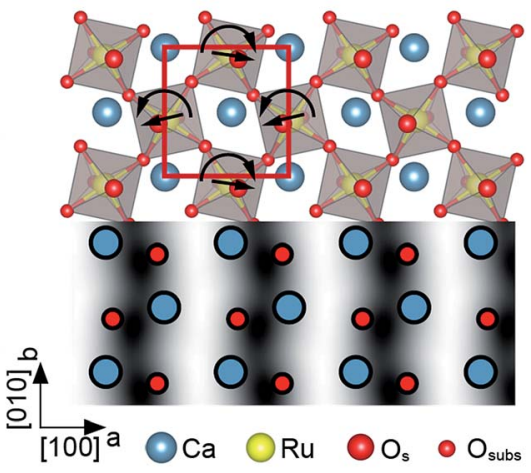

d

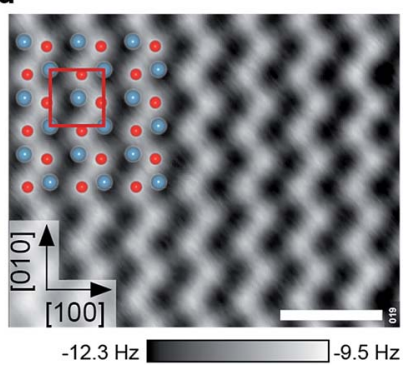

Fig. 1 The calcium ruthenate structure. All scale bars correspond to $1 \mathrm{~nm}$. (a) Unit cell of the $n=2$ member of the $\mathrm{Ca}_{n+1} \mathrm{Ru}_{n} \mathrm{O}_{3 n+1}$ Ruddlesden Popper series. Cleavage planes are marked in gray. (b) Top view and STM simulation (Tersoff-Hamann approximation ${ }^{40}$ ) of the $\mathrm{CaO}$-terminated $\mathrm{Ca}_{3} \mathrm{Ru}_{2} \mathrm{O}_{7}(001)$ surface. The $\mathrm{RuO}_{6}$ octahedra are alternately tilted with respect to the $c$ axis (most pronounced in the ac plane), and rotated in the ab plane as indicated by the straight and curved arrows, respectively. The red box marks the orthorhombic unit cell ( $a=5.365 \AA, b=5.562 \AA$ ). (c) STM image of the cleaved surface. The dark (bright) substrate lines in [010] direction correspond to areas where the apical oxygen atoms of the $\mathrm{RuO}_{6}$ octahedra are tilted towards (away from) each other, compare to (b). The point defects are attributed to spurious impurities (see ESI $\dagger$ ). STM parameters: $V_{\text {sample }}=$ $+0.8 \mathrm{~V}, I_{\text {tunnel }}=0.1 \mathrm{nA}, T_{\text {sample }}=78 \mathrm{~K}$; image rotated and cropped; fast scan direction is $68^{\circ}$ clockwise from horizontal. (d) Constant-height nc-AFM image, different area than in (c). Shown is the frequency shift, $\Delta f$. The apical oxygen atoms are imaged as bright dots (less negative $\Delta f$, more repulsive), arranged in zig-zag lines in [010] direction. AFM parameters: $A=100 \mathrm{pm}, V_{\text {sample }}=+0.25 \mathrm{~V}, T_{\text {sample }}=4.8 \mathrm{~K}$; fast scan direction is $50^{\circ}$ clockwise from horizontal.

a cleaving energy of $3.62 \mathrm{eV}$ (DFT value) per unit cell. This low cleaving energy is consistent with experiments, where only the $\mathrm{CaO}$ termination is consistently observed upon cleaving single crystals in UHV. These $\mathrm{CaO}$ terraces are typically at least a few hundred nm in size.

The tilt and rotation of the octahedra leads to a distorted surface oxygen sublattice that was found essential in affecting the ordering of a hydroxyl overlayer on this surface. ${ }^{29}$ STM shows alternating bright and dark lines along the [010] direction, see Fig. 1c. According to a STM simulation (TersoffHamann approximation) ${ }^{40}$ the dark (bright) substrate lines correspond to areas where the apical oxygen atoms of the $\mathrm{RuO}_{6}$ octahedra are tilted towards (away from) each other (Fig. 1b). All non-contact atomic force microscopy (nc-AFM) images in this work show the frequency shift, $\Delta f$, and are recorded in constantheight mode, where darker color means higher attractive force.

Pioneering works on oxide surfaces show that the AFM contrast is governed by the electric charge of the tip apex. ${ }^{41,42}$ 'Positively terminated' tips interact attractively with anions, and repulsively with cations. 'Negative tips' show the opposite behaviour. The contrast in Fig. 1d corresponds to the negative termination; simultaneously measured STM and AFM images show that the apical oxygen atoms interact repulsively with the tip and thus are imaged as bright dots arranged in zig-zag lines in [010] direction.

The observed point defects are attributed to impurities (Ti, $\mathrm{Sr}, \mathrm{Mg}, \mathrm{Ba}$ ) in the material (see Table $\mathrm{S} 1 \dagger$ ) rather than intrinsic defects that would stem from the cleaving process. The calculated creation energies for $\mathrm{O}$ and Ca vacancies, 3.9 and $5.3 \mathrm{eV}$, respectively, are significantly higher than the cleaving energy per broken Ca-O bond of $0.91 \mathrm{eV}$. Also, the appearance in STM of $\mathrm{O}$ vacancies created by electron irradiation is known from previous work. $^{29}$

\section{Low coverage of $\mathrm{O}_{2}^{-}$}

The theoretical description of complex oxides is challenging and the currently-available approximations deliver only incomplete descriptions. Here results from DFT calculations including van-der-Waals interactions (vdW-DFT) are discussed first, for a more detailed analysis using 'beyond-DFT' methods, see section 'Electronic Properties'. The vdW-DFT calculations predict adsorption of $\mathrm{O}_{2}$ as a charged molecule with an adsorption energy of $1.4 \mathrm{eV}$. The molecule adsorbs as a superoxo species at a Ca-Ca bridge, labelled B1 in Fig. 2a. The octahedral tilt results in two non-equivalent bridge sites, the one chosen by the molecule (B1, equivalent to B2) lies on the bright substrate lines in STM images (Fig. 1b). Due to the charge transfer, the intramolecular $\mathrm{O}-\mathrm{O}$ bond length is increased to a value of $1.35 \AA$. The molecule is rotated by $34^{\circ}$ with respect to the bright substrate lines, and tilted by $29^{\circ}$ with respect to the surface plane, see Fig. $2 \mathrm{~b}$. One $\mathrm{O}$ of the molecule is located almost at the center of the $\mathrm{Ca}-\mathrm{Ca}$ bridge $(\mathrm{O}-\mathrm{Ca} 1=2.37 \AA, \mathrm{O}-\mathrm{Ca} 2=2.46 \AA)$, the other $\mathrm{O}$ sits far away from $\mathrm{Ca} 1(\mathrm{O}-\mathrm{Ca} 1=3.69 \AA)$, but maintains a similar distance to $\mathrm{Ca} 2(\mathrm{O}-\mathrm{Ca} 2=2.34 \AA)$ as the first
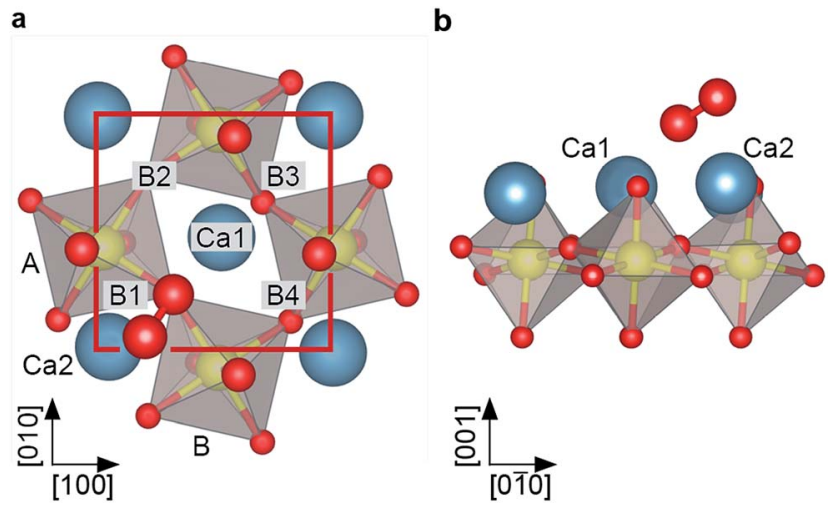

Fig. 2 DFT model of $\mathrm{O}_{2}{ }^{-}$adsorbed on $\mathrm{Ca}_{3} \mathrm{Ru}_{2} \mathrm{O}_{7}$. (a) Top view and (b) side view of the vdW-DFT model for $\mathrm{O}_{2}{ }^{-}$with $E_{\text {ads }}=1.4 \mathrm{eV}$. The molecule adsorbs as a tilted superoxo species close to a Ca-Ca bridge (here B1) 
$\mathrm{O}$ due to the tilt of the molecule. The molecule slightly distorts the substrate as the tilt of the two nearest $\mathrm{RuO}_{6}$ octahedra is increased to $15^{\circ}$ compared to $13^{\circ}$ in the pristine surface. In addition, the volume of these octahedra decreases by $1.64 \%$ and $0.26 \%$, respectively, due to a shortening of the $\mathrm{Ru}-\mathrm{O}_{\text {surf }}$ bond. Adsorption at B3 or B4, i.e., at positions where STM shows a dark line, is disfavoured by $0.4 \mathrm{eV}$ due to the shorter distances to the surface oxygen atoms and the resulting repulsion between $\mathrm{O}_{\text {surf }}^{2-}$ and $\left(\mathrm{O}_{2}{ }^{-}\right)$ads. Dissociative adsorption results in an energy penalty close to $1 \mathrm{eV}$.

When the $\mathrm{Ca}_{3} \mathrm{Ru}_{2} \mathrm{O}_{7}(001)$ surface is exposed to a low dose of $\mathrm{O}_{2}$ at $5 \mathrm{~K}$, AFM images taken at $0 \mathrm{~V}$ sample bias voltage show dark spots of varying contrast and size, see Fig. 3a. When applying a slightly negative or positive sample bias voltage, tunnelling current starts to flow and a few adsorbates interact with the tip, see Fig. 3b. In Fig. 3c two larger, dark spots change their position and contrast in the consecutive image in Fig. 3d and an additional feature appears in the vicinity of the two spots. This suggests that the varying contrast and size of the dark spots at zero bias voltage (Fig. 3a) can be attributed to molecules adsorbed at surface defects or in non-equilibrium positions, or molecules adsorbed too close to each other to be resolved.

It is well-known that the tip's electric field and/or the tunnelling current can influence adsorbates. ${ }^{\mathbf{4 3 , 4 4}}$ The calculated $\mathrm{O}-\mathrm{O}$ stretch mode for the adsorbed $\mathrm{O}_{2}{ }^{-}$lies at $139 \mathrm{meV}$
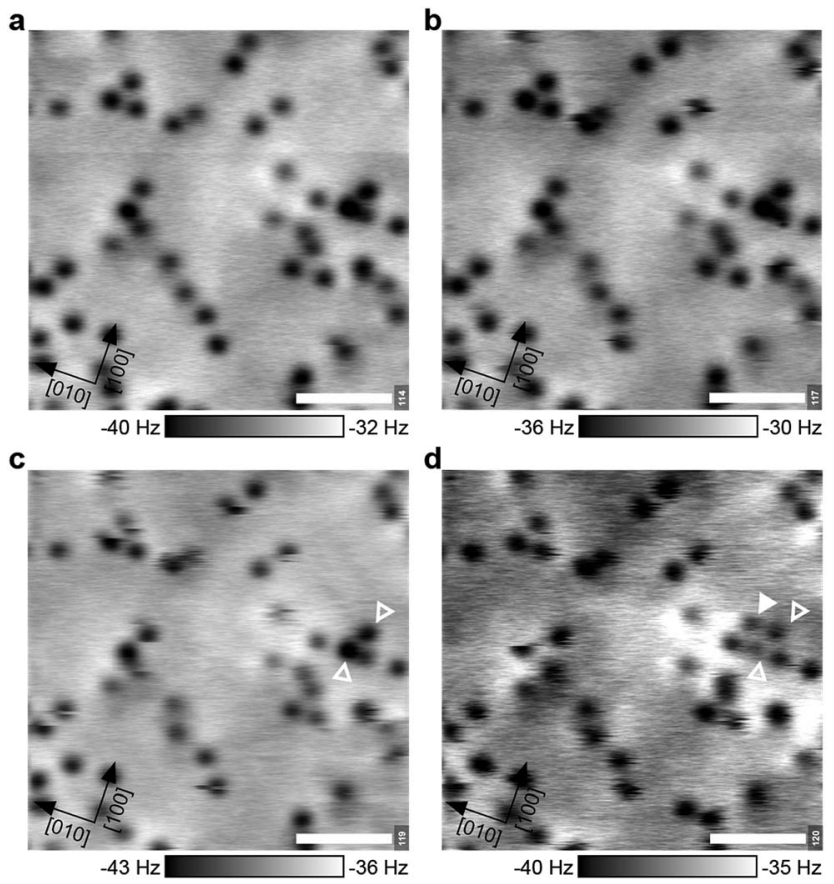

Fig. 3 Adsorbed $\mathrm{O}_{2}{ }^{-}$in constant-height nc-AFM. Molecular oxygen was dosed at $5.5 \mathrm{~K}$. All panels show the same area (common AFM parameters: $A=40 \mathrm{pm}, T_{\text {sample }}=4.8 \mathrm{~K}$, scale bars $2 \mathrm{~nm}$ ) but varying bias voltages $V_{\text {sample }}$ and tip-sample distances $\Delta z$. (a) $V_{\text {sample }}=0 \mathrm{~V}$. (b) $V_{\text {sample }}=-120 \mathrm{mV}, \Delta z=+25 \mathrm{pm}$. (c) $V_{\text {sample }}=+150 \mathrm{mV}, \Delta z=0 \mathrm{pm}$. (d) $V_{\text {sample }}=+220 \mathrm{mV}, \Delta z=+28 \mathrm{pm}$. Open triangles in (c, d) mark the same position. The full triangle marks a new feature, suggesting that the darker spots contain more than one molecule.
(1121 $\mathrm{cm}^{-1}$ ); exciting this mode could be the reason for the increase in mobility observed in Fig. 3c, d. In comparison, STM images of a similarly-prepared sample taken at $78 \mathrm{~K}$ show only streaks across surface defects, see Fig. 4. Occasionally the streaks continue along the [010] direction after the tip first scanned across a defect, indicating that a molecule was being moved along the bright substrate line. This shows that the molecules first adsorb at surface defects and the difficulty of imaging weakly-bound adsorbates with STM, see also Fig. S1. $\dagger$

\section{High coverage of $\mathrm{O}_{2}$}

At a higher coverage of one molecule per primitive unit cell, DFT calculations predict adsorption in a zig-zag arrangement, where all $\mathrm{O}_{2}{ }^{-}$are spaced one lattice constant apart in [010] direction and adjacent rows are shifted by half a lattice constant in [010] direction, see Fig. 5. With the $\mathrm{O}_{2}{ }^{-}$in one row adsorbed exclusively at B1 and in the adjacent one adsorbed exclusively at B2, they form a $(2 \times 1)$ overlayer. Although the $\mathrm{O}_{2}{ }^{-}$is still adsorbed as an intact superoxide ion with bond length $1.35 \AA$ and a vibrational stretching frequency of $1121 \mathrm{~cm}^{-1}$, the vdW-DFT adsorption energy is reduced to $1.19 \mathrm{eV}$ per $\mathrm{O}_{2}{ }^{-}$. This decrease of approximately $0.2 \mathrm{eV}$ is mainly attributed to the electrostatic repulsion, as the minimal distance between the $\mathrm{O}_{2}{ }^{-}$molecules is decreased from $16 \AA$ to $6 \AA$. The $\mathrm{O}_{2}{ }^{-}$are located on the bright substrate lines, between a $\mathrm{Ca}$ and an apical $\mathrm{O}$ atom. In contrast to the low-coverage case the adsorbate is only slightly rotated azimuthally to the [010] direction; tilted by just $4^{\circ}$ with respect to the surface plane. The tilting of the two $\mathrm{RuO}_{6}$ closest to the adsorbate is $13.4^{\circ}$, slightly more than for the pristine surface $\left(13.0^{\circ}\right)$. A parallel arrangement, where adsorbed $\mathrm{O}_{2}{ }^{-}$in adjacent rows are not shifted by half a lattice constant in

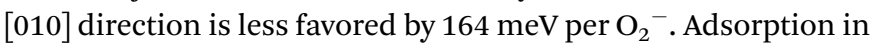
$\mathrm{B} 3$ or B4, i.e. the dark substrate line, is disfavored by $448 \mathrm{meV}$, similar to the low-coverage case.

The STM simulation of the $(2 \times 1)$ overlayer shows bright, elongated spots corresponding to a superposition of two lobes of the neighboring $\mathrm{O}_{2}{ }^{-}$, separated by a dark node at the center

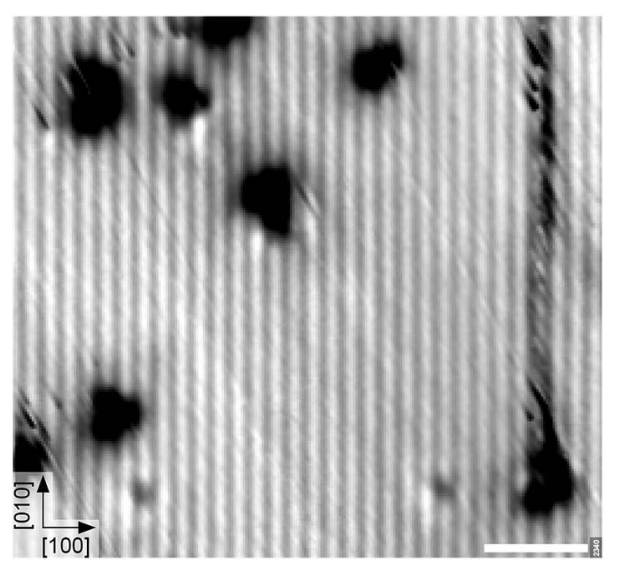

Fig. 4 STM image after dosing $0.025 \mathrm{~L} \mathrm{O}_{2}$ at $110 \mathrm{~K}$. The molecules appear as streaks across point defects. STM parameters: $V_{\text {sample }}=$ $+1.0 \mathrm{~V}, I_{\text {tunnel }}=0.1 \mathrm{nA}, T_{\text {sample }}=78 \mathrm{~K}$; fast scan direction is $58^{\circ}$ clockwise from horizontal. The scale bar corresponds to $3 \mathrm{~nm}$. 
a

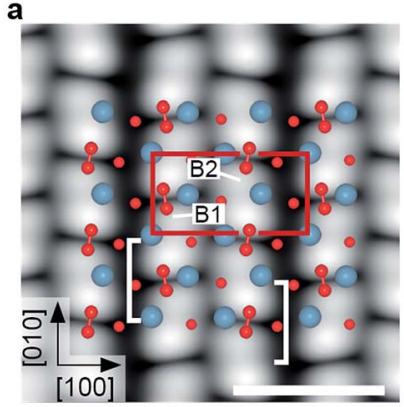

c

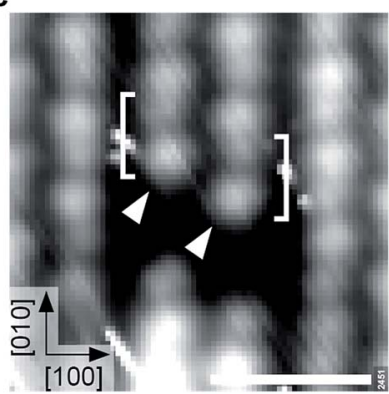

b

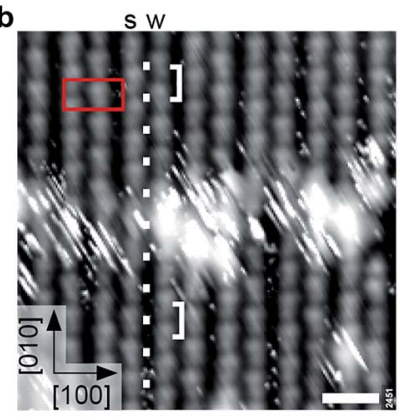

d

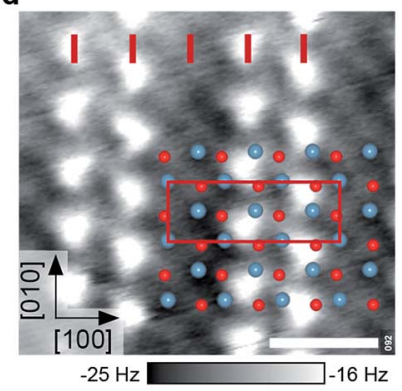

Fig. 5 Ordered superoxide overlayers. All scale bars correspond to $1 \mathrm{~nm}$. (a) DFT model and STM simulation $\left(V_{\text {sample }}=+0.8 \mathrm{~V}\right)$ of the $(2 \times$ 1) overlayer with one molecule per primitive unit cell. Bridge sites $B 1$ and $\mathrm{B} 2$ are labeled as in Fig. 2. Note that the $\mathrm{O}_{2}{ }^{-}$appear as bright elongated spots with a dark node in the center (brackets). (b) Experimental STM image of the $(2 \times 1)$ overlayer. The bright, streaky area at the center is a domain boundary as indicated by the registry of the white dots. The $\mathrm{O}_{2}{ }^{-}$rows show a weak ('w') or strong ('s') degree of undulation. (STM parameters: $V_{\text {sample }}=+1.0 \mathrm{~V}, I_{\text {tunnel }}=0.05 \mathrm{nA}, T_{\text {sample }}$ $=78 \mathrm{~K}$; fast scan direction is $59^{\circ}$ clockwise from horizontal.) (c) Detailed STM image (same parameters) of $\mathrm{O}_{2}{ }^{-}$adsorbed next to a surface defect. Both rows begin with a bright spot (arrows) followed by the distinct node and a slightly weaker spot, in agreement with (a). (d) AFM image of a partially covered sample, showing a $(3 \times 1)$ overlayer. Red ticks mark lattice-constant intervals along [100]. The $\mathrm{O}_{2}{ }^{-}$and surface $\mathrm{O}$ are imaged as intense and faint, bright spots, respectively (corresponding to repulsion). AFM parameters: $A=$ $500 \mathrm{pm}, V_{\text {sample }}=0 \mathrm{~V}, T_{\text {sample }}=4.8 \mathrm{~K}$.

of the molecular bond, rather than between two molecules (see Fig. 5a). The electronic contrast leads to an apparently alternating spacing of the rows in STM although the $\mathrm{O}_{2}{ }^{-}$ molecules are equally spaced in [100] direction. Here only a STM simulation is shown as the simulation of atomically-resolved nc-AFM is more involved, since the tip has to be taken into account explicitly.

When going from low coverage towards saturation, the molecules become less mobile and STM images less streaky, until the $(2 \times 1)$ overlayer forms, see Fig. S1. $\dagger$ The experimental saturation coverage of close to one monolayer $(\mathrm{ML}$, where $1 \mathrm{ML}$ is defined as one adsorbate per primitive unit cell) is shown in Fig. 5b. The $\mathrm{O}_{2}{ }^{-}$covered part of the surface appears as rows of pairs of a bright and a slightly weaker spot on a black background, running in [010] direction. The distance between the pairs along the row is one lattice constant, and every other gap between the bright and slightly weaker spots is more pronounced. Neighbouring rows are shifted by half a lattice constant in [010] direction, and appear subtly different as the

spots in one row strongly (weakly) undulate with respect to the [100] direction, marked by $\mathrm{s}(\mathrm{w})$ in Fig. 5b. The $\mathrm{O}_{2}{ }^{-}$rows have alternatingly narrow and wide gaps in [100] direction. The bright, streaky areas are domain boundaries: the spacing of the rows labelled $\mathrm{s}$ and $\mathrm{w}$ is narrow in the upper half of the image, but wide in the lower half. The row that undulates strongly in the upper half of the image undulates weakly in the lower half and vice versa. This is because the molecules are shifted half a lattice constant in [010] direction across the domain boundary, where the row with all $\mathrm{O}_{2}{ }^{-}$adsorbed in $\mathrm{B} 1$ sites becomes a row with all $\mathrm{O}_{2}{ }^{-}$adsorbed in $\mathrm{B} 2$ sites and vice versa (compare to the white dots that mark lattice-constant-intervals along [010]). The streaky appearance of the domain boundaries indicates weakly-bound molecules that cannot be imaged with STM. This results in an apparent coverage of only 0.9 ML when counting all $\mathrm{O}_{2}{ }^{-}$on the rows.

Fig. 5c shows two $\mathrm{O}_{2}{ }^{-}$rows (marked by arrows) that start next to an unknown, dark surface defect. Both rows begin with a bright spot followed by the more pronounced gap and a slightly weaker spot. This agrees well with the STM simulation showing a distinct node in the center of the molecules, and a weaker depression between two molecules. To confirm that the $\mathrm{O}_{2}{ }^{-}$are adsorbed in either B1 or B2 sites, i.e., on the bright substrate lines, a part of the overlayer was removed by scanning with higher bias voltage $(+2.0 \mathrm{~V})$, see Fig. S2. $\dagger$

While, as pointed out above, STM disturbs molecules at low coverage too much for reliable imaging, this is possible with AFM. Fig. 5d shows an AFM image of a sample partially covered with $\mathrm{O}_{2}{ }^{-}$. It should be noted that after the tip approach with non-zero sample bias voltage the tip was moved to a different region with $0 \mathrm{~V}$ bias voltage to exclude a possible charging or manipulation of the adsorbate upon the initial scan. In AFM the molecules are imaged as bright spots (less negative $\Delta f$, more repulsive). The surface oxygen atoms of the uncovered sample between the $\mathrm{O}_{2}{ }^{-}$rows are imaged as faint, bright spots. The overlay shows the surface atoms of the pristine surface aligned to the uncovered part of the sample, and confirms that the $\mathrm{O}_{2}{ }^{-}$ molecules adsorb close to a Ca-Ca bridge. In contrast to STM images the rows are equally spaced by one lattice constant in [100] direction. Thus the alternating row spacing in STM images (Fig. 5b) is mainly due to a difference in electronic contrast.

\section{Electronic properties}

The $\mathrm{Ca}_{3} \mathrm{Ru}_{2} \mathrm{O}_{7}$ substrate shows a first-order transition from a metallic to a non-metallic phase at $48 \mathrm{~K}^{45}$ This is reflected in the theoretical description: while the standard vdW-DFT calculations yield the metallic ground state due to the common underestimation of the electronic band gap, more involved approaches such as hybrid functional (HSE06) calculations predict the formation of a gap of $1.27 \mathrm{eV}$. A single iteration of many-electron $\left(\mathrm{G}_{0} \mathrm{~W}_{0}\right)$ corrections ${ }^{33}$ does not qualitatively change this finding.

A detailed analysis of the electronic properties of the $(2 \times 1)$ overlayer shows that the $\mathrm{O}_{2}$ molecule does not adsorb as a neutral molecule. The density of states show an additional, occupied $\pi^{*}$ state in the minority channel, leading to the 
formation of a charged $\mathrm{O}_{2}{ }^{-}$species, regardless of the level of theory, see Fig. 6 . An analysis of the differential charge shows that the electron is transferred into the $\pi^{*}$ orbital oriented towards the nearest Ca, filling this orbital, see Fig. 7a, b. This is also reflected in the magnetic properties: the neutral gas phase $\mathrm{O}_{2}$ molecule has two half-filled $\pi^{*}$ orbitals in the triplet state resulting in a magnetic moment of $2 \mu_{\mathrm{B}}$. Upon adsorption, one $\pi^{*}$ orbital becomes filled, leaving the second orthogonal $\pi^{*}$ state singly occupied. This orbital carries the remaining magnetic moment of about $1 \mu_{\mathrm{B}}$ (see Fig. 7c). The electron is transferred from the RuO states at the Fermi level in case of the metallic substrate phase described by vdW-DFT, and from the $\mathrm{Ru}$ of the closest $\mathrm{RuO}_{6}$ octahedron in the insulating substrate phase described by the HSE06 functional. As a consequence of the more localized electron transfer in the insulating phase the $\mathrm{RuO}_{6}$ octahedra in the surface layer closest to the $\mathrm{O}_{2}{ }^{-}$molecule contract by $5.2 \%$ while the remaining two expand by $3.0 \%$, see Fig. S3a. $\dagger$ In vdW-DFT approximation, only a slight contraction $(3 \%)$ of the octahedra close to the molecule takes place (Fig. S3b $\dagger$ ), where the bond between the central $\mathrm{Ru}$ and the apical $\mathrm{O}$ atom is shortened. Compared to the metallic phase, only minor structural changes occur in the HSE06 calculations: the $\mathrm{O}_{2}{ }^{-}$molecules rotate by $4.5^{\circ}$ clockwise and $4.3^{\circ}$ counterclockwise, and the tilting angle is increased to a value of $12^{\circ}$ with respect to the surface plane. Although the $\mathrm{O}_{2}{ }^{-}$adsorption geometries are almost identical, the adsorption energy decreases from $1.19 \mathrm{eV}$ to $0.58 \mathrm{eV}$ and the HOMO-LUMO gap of the $\mathrm{O}_{2}{ }^{-}$increases from $0.7 \mathrm{eV}$ to $3.1 \mathrm{eV}$ when comparing the HSE06 to the vdW-DFT result. The HSE06 functional certainly improves the description of the electronic structure of the

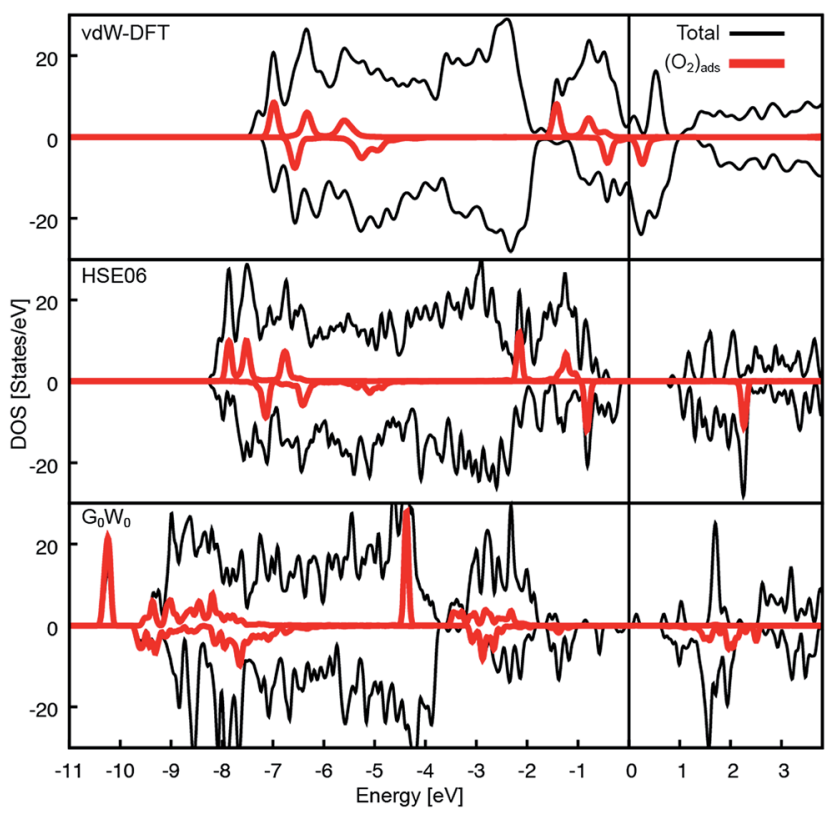

Fig. 6 Density of states for the $(2 \times 1)$ overlayer. Total and $\mathrm{O}_{2}$ DOS for the different theoretical approaches. In all cases charge is transferred into the split $\pi^{*}$ states of the $\mathrm{O}_{2}$ in the spin down channel. The HOMO-LUMO gap is 0.7, 3.1 and approximately $1.7 \mathrm{eV}$ on the DFT, HSE06 (insulating phase) and $G_{0} W_{0}$ level, respectively.

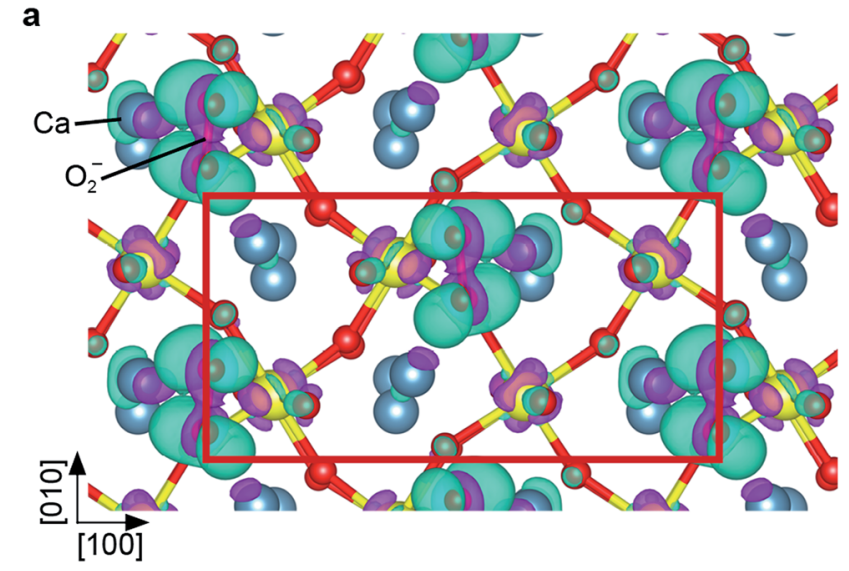

b

C
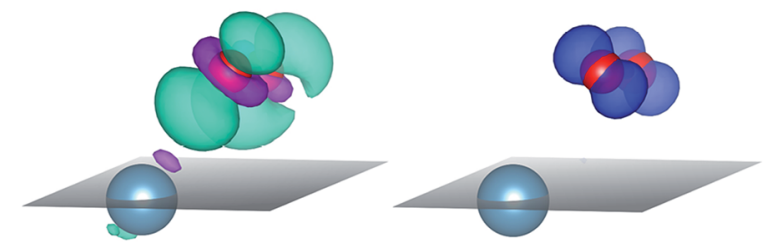

Fig. 7 (a) Charge density difference plot of the $(2 \times 1)$ overlayer, top view: charge is transferred to and from the turquoise and violet areas, respectively. (b) Detailed view (surface plane marked in grey) of the charge difference of the $\mathrm{Ca}$ and $\mathrm{O}_{2}{ }^{-}$labelled in (a): the additional electron is transferred into a $\pi^{*}$ state rotated towards the nearest $\mathrm{Ca}$ atom. (c) Magnetization density plot: the blue colored orbitals carry the magnetic moment of the superoxo species. These orbitals are perpendicular to the orbital that the additional electron is transferred to, compare to (b) (same perspective).

molecular adsorbate, but does not capture the metallic character of the substrate and tends to underestimate the screening properties of metals and small-gap semiconductors. ${ }^{46}$ However, the HSE06 adsorption energy of $0.58 \mathrm{eV}$ agrees well with the experimental estimate of $0.6 \mathrm{eV}$ (see below).

To assess why the adsorption energy according to vdW-DFT is too high compared to the experimental value, and whether the strong decrease in adsorption energy in the HSE06 calculation is due to the insulating substrate phase or the increased HOMO-LUMO gap, a state-of-the-art many-electron-approach in the random phase approximation ${ }^{47}$ (RPA) was used. The RPA calculations predict an $\mathrm{O}_{2}{ }^{-}$adsorption energy of $0.72 \mathrm{eV}$ and the corresponding $\mathrm{G}_{0} \mathrm{~W}_{0}$ calculations result in the metallic substrate phase with a HOMO-LUMO gap of the $\mathrm{O}_{2}{ }^{-}$of approximately $1.7 \mathrm{eV}$ (see Fig. 6c), which is significantly larger compared to the vdW-DFT calculation. The increase in the adsorption energy of $0.14 \mathrm{eV}$ compared to HSE06 is attributed to additional non-local contributions such as vdW interactions that are not considered in HSE06.

The comparison of different theoretical approaches (see Table 1) indicates several contributions to the adsorption energy: comparing the $\mathrm{PBE}$ to the $\mathrm{PBE}+\mathrm{U}$ results shows that the overestimation of the polarizability of the surface on the GGA level contributes about $0.2 \mathrm{eV}$, a similar increase as obtained by including vdW corrections. A third contribution stems from the underestimation of the HOMO-LUMO gap: since the charge 
Table 1 DFT adsorption energies of $\mathrm{O}_{2}^{-}$on $\mathrm{Ca}_{3} \mathrm{Ru}_{2} \mathrm{O}_{7}(001)$. Adsorption energy per molecule of the $(2 \times 1)$ overlayer obtained at different levels of theory. The insulating phase was modelled by an additional on-site interaction ${ }^{50}(U-J=4 \mathrm{eV})$ added to the Ru d-states. The vdW contributions amount to about $0.27 \mathrm{eV}$

\begin{tabular}{ll}
\hline Computational method & Adsorption energy $[\mathrm{eV}]$ \\
\hline PBE & 0.93 \\
PBE+U $(U-J=4 \mathrm{eV})$ & 0.68 \\
vdW-DF & 1.19 \\
HSE06 & 0.59 \\
RPA & 0.72
\end{tabular}

state of the molecule changes, a correct description of the electron affinity, i.e., the cost to add an additional electron to the $\mathrm{O}_{2}$ molecule, plays a key role for the energy balance. Comparing the PBE and the HSE06 functional, advanced quantum-chemical (CCSD $(\mathrm{T})$ ) calculations ${ }^{\mathbf{4 8 , 4 9}}$ show an electron affinity difference of gas phase $\mathrm{O}_{2}$ of $0.15 \mathrm{eV}$, which is close to the predicted difference in adsorption energy when comparing the DFT+U and the HSE06 results.

\section{Spectroscopies on the $(2 \times 1)$ overlayer}

To gain experimental information on the charge state of the $\mathrm{O}_{2}$ overlayer, point Kelvin probe spectroscopy ${ }^{51}$ was performed. For a quantitative analysis it was assumed that the $\mathrm{Cu}(110)$ sample used for tip preparation has a work function $(\Phi)$ of $4.5 \mathrm{eV} .^{52}$ The measured local contact potential difference (LCPD) of the $\mathrm{Cu}$ sample of $+0.4 \mathrm{~V}$ gives a tip $\Phi$ of $4.1 \mathrm{eV}$, according to $V_{\mathrm{LCPD}}=$ $\left(\Phi_{\text {sample }}-\Phi_{\text {tip }}\right) /|e|$, with $e$ the charge of the electron. This tip was then used to measure the LCPD on a sample where part of the $(2 \times 1)$ overlayer was removed (similar to one shown in Fig. $S 2 \dagger)$ allowing to measure on the $(2 \times 1)$ overlayer and the pristine surface in close succession, thus excluding a tip change between the measurements. LCPD values of $-1.45 \mathrm{~V}$ and $+0.2 \mathrm{~V}$ were found for the pristine surface and the $(2 \times 1)$ overlayer, respectively, see Fig. 8a. Combining the known tip $\Phi$ and the measured LCPDs gives a $\Phi$ of 2.7 and $4.3 \mathrm{eV}$ for the pristine surface and the $(2 \times 1)$ overlayer, respectively. Such a work function increase is due to a negatively charged adsorbate overlayer. ${ }^{53}$ DFT calculations give a $\Phi$ of $3.1 \mathrm{eV}$ and $5.4 \mathrm{eV}$ for the pristine surface and the $(2 \times 1)$ overlayer, respectively.

In X-ray photoelectron spectroscopy (XPS) the pristine surface exhibits an asymmetric O 1s peak at $529.1 \mathrm{eV}$ binding energy (BE), broadened towards the high BE side, see Fig. 8b. According to vdW-DFT calculations the $\mathrm{O} 1 \mathrm{~s}$ core level for the $\mathrm{O}$ atoms in the rock-salt like $\mathrm{CaO}$ planes is shifted to $0.7 \mathrm{eV}$ higher $\mathrm{BE}$ compared to the $\mathrm{RuO}_{2}$ planes, which serve as DFT reference. On a structurally related $\mathrm{Sr}_{2} \mathrm{RuO}_{4}$ sample, ${ }^{28}$ two distinct $\mathrm{O}$ 1s peaks were observed and attributed to the apical and equatorial $\mathrm{O}$ atoms. The observation of only one asymmetric peak for $\mathrm{Ca}_{3} \mathrm{Ru}_{2} \mathrm{O}_{7}$ is attributed to the limited resolution of the experimental setup, the $3: 4$ ratio of $\mathrm{O}$ atoms in the $\mathrm{CaO}$ and $\mathrm{RuO}_{2}$ planes, and potentially the different electronic structure of these materials. Exposing the sample to $2.5 \mathrm{~L} \mathrm{O}_{2}$ at $110 \mathrm{~K}$ results in a $(2 \times 1)$ overlayer and an $\mathrm{O} 1 \mathrm{~s}$ peak at $532.6 \mathrm{eV}$ BE; i.e. $3.5 \mathrm{eV}$
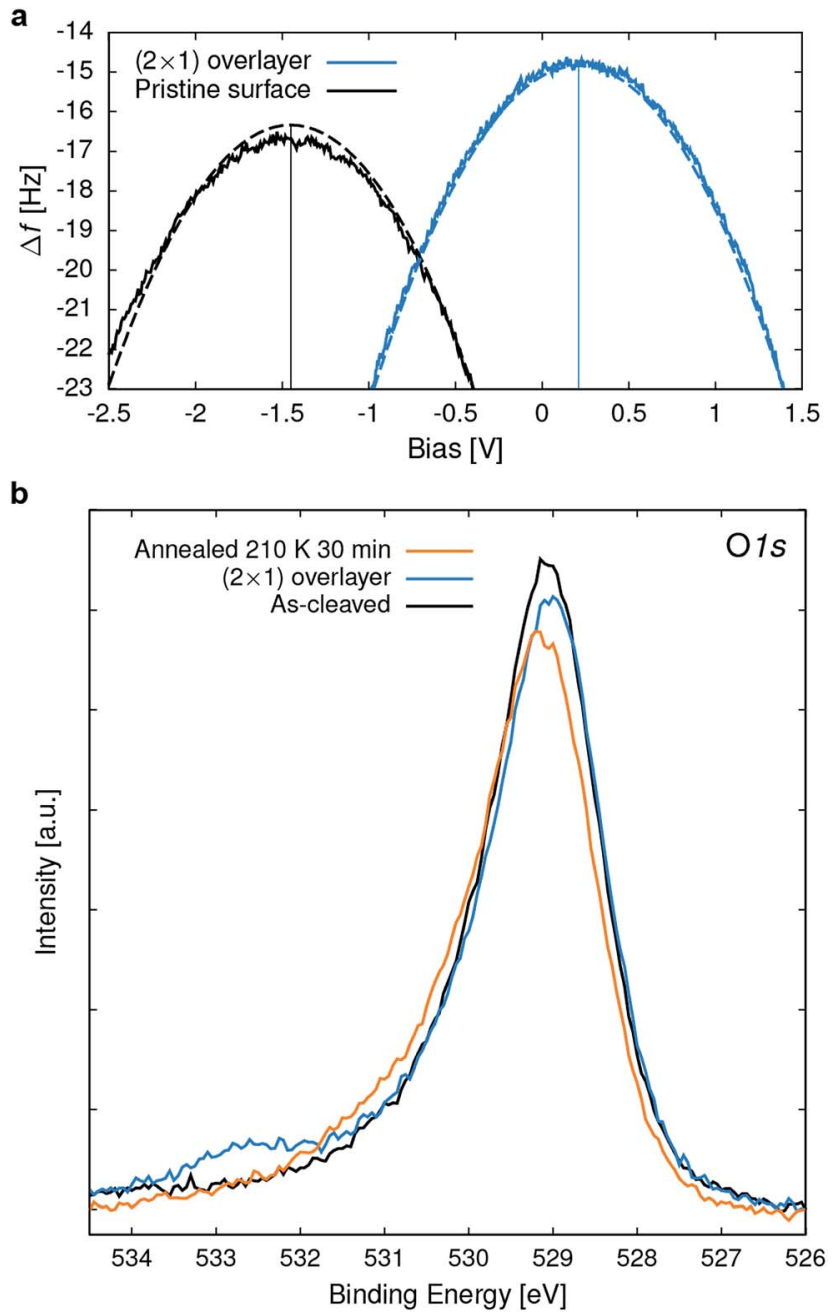

Fig. 8 Surface spectroscopies. (a) Point Kelvin probe spectroscopy of the pristine surface and the $(2 \times 1) \mathrm{O}_{2}{ }^{-}$overlayer at $78 \mathrm{~K}$. Shown are the measured (full line) and the numerically fitted (dashed lines) Kelvin parabolas, reflecting a change in work function (see main text for analysis details). (b) $\bigcirc 1 \mathrm{~s}$ XPS ( $\mathrm{Mg} \mathrm{K} \alpha, T_{\text {sample }}=110 \mathrm{~K}$ ) spectra of the ascleaved surface, the $(2 \times 1) \mathrm{O}_{2}^{-}$overlayer and after annealing at $210 \mathrm{~K}$ for $30 \mathrm{~min}$.

above the peak of the pristine surface. In addition, the lattice oxygen peak shifts towards lower BE due to an upward band bending of $0.1 \mathrm{eV}$. The $\mathrm{O}_{2}{ }^{-}$peak lies approximately half way between reported BEs for physisorbed, neutral $\mathrm{O}_{2}$ of 536 to $538 \mathrm{eV}$ (ref. 9 and 54) and the double negatively charged bulk $\mathrm{O}$ at $529.1 \mathrm{eV}$. Thus this XPS result is also consistent with a superoxo species. The calculation for the adsorbed $\mathrm{O}_{2}{ }^{-}$gives an initial-state shift of $1.3 \mathrm{eV}$ (which would result in $530.4 \mathrm{eV}$ $\mathrm{BE}$ ), which is significantly lower than the experimental peak at $532.6 \mathrm{eV}$. Calculations including the final state approximation have been attempted but were ultimately not considered, as they resulted in an unphysical over-screening of the core-hole: the unoccupied $\mathrm{O}_{2} \pi^{*}$ orbital calculated just above the Fermi level is pulled below it and localizes an additional electron at the molecule, which directly leads to an underestimation of the binding energy. ${ }^{55}$ While using a hybrid functional approach (such as HSE06) partially cures this problem by shifting the 
unoccupied $\mathrm{O}_{2} \pi^{*}$ orbital away from the Fermi level, it also leads to an insulating substrate and therefore was not considered for the evaluation of the core level shifts.

For the superoxo species a double peak with a $1: 3$ ratio is expected due to the coupling of the $\mathrm{O}_{1 \mathrm{~s}}$ hole spin to the $\mathrm{O}_{2 \mathrm{p}}$ valence states resulting in a single or triplet final state configuration. Similarly, adsorbed neutral $\mathrm{O}_{2}$ leads to a double peak with a $2: 4$ ratio (doublet and quadruplet) and a splitting of roughly $1 \mathrm{eV} .^{9,54}$ The observation of only one broad peak in Fig. $8 \mathrm{~b}$ is attributed to the relatively small splitting of $0.25 \mathrm{eV}$ (estimated from DFT calculations) and the limited experimental resolution.

When annealing the $\mathrm{O}_{2}{ }^{-}$saturated sample to room temperature, the molecule desorbs, the band bending vanishes, and subsequent STM images show the pristine surface. The superoxo peak in the $O$ 1s XPS starts to disappear at approximately $200 \mathrm{~K}$, see Fig. 8b. The slight increase at higher binding energy is probably due to hydroxyls that accumulated during this experiment. ${ }^{29}$ A rough estimate for the adsorption energy based on the desorption temperature is $0.6 \mathrm{eV}$, which is in good agreement with the calculated adsorption energy of $0.72 \mathrm{eV}$ (RPA).

\section{Discussion}

These results provide clear evidence of the adsorption geometry and electronic structure of $\mathrm{O}_{2}{ }^{-}$on a well-defined perovskite-type surface and it is interesting to draw comparisons to studies on metals and binary metal oxides.

The spectroscopic results and DFT calculations conclusively show that molecular oxygen readily adsorbs as $\mathrm{O}_{2}{ }^{-}$on the ascleaved $\mathrm{Ca}_{3} \mathrm{Ru}_{2} \mathrm{O}_{7}(001)$ surface. The calculated bond length of $1.35 \AA$ and the internal stretching frequency of $1121 \mathrm{~cm}^{-1}$ agrees well to the values found for $\mathrm{O}_{2}{ }^{-}$on late transition metals. ${ }^{56}$ The source of electrons for charging the $\mathrm{O}_{2}$ are the $\mathrm{Ru}$ d-states in the $\mathrm{RuO}_{2}$ subsurface layer below the CaO-terminated surface. According to the calculations, in the metallic phase the electron is transferred from the valence band, while in the insulating phase it is transferred mainly from a neighboring $\mathrm{RuO}_{6}$ octahedron, which is also reflected in a stronger contraction of that octahedron (see Fig. S3a $\dagger$ ). This charge transfer is crucial for the adsorption: on $\mathrm{CaO}(001)$ chemisorption is only observed after introducing Mo impurities that donate electrons to the oxide, ${ }^{18}$ while the $\mathrm{RuO}_{2}$ layers in $\mathrm{Ca}_{3} \mathrm{Ru}_{2} \mathrm{O}_{7}$ can be seen as intrinsic electron donors. Consequently a full monolayer of adsorbed $\mathrm{O}_{2}{ }^{-}$can form. The transfer of a second electron, i.e., the formation of a peroxo species, is hindered by a too weak hybridization of the states of the substrate and the molecule. This is supported by the density of states that shows that the remaining unoccupied $\pi^{*}$ state is located substantially above $E_{\mathrm{F}}$ (Fig. 6).

In an earlier study it was found that $\mathrm{O}_{2}$ adsorbs as a neutral species on defect-free $\mathrm{SrTiO}_{3}(001),{ }^{23}$ but as an activated species on $\mathrm{La}_{2} \mathrm{NiO}_{4}(001){ }^{24}$ This observation was correlated to the charge transferred from the A-site ions: ${ }^{24}$ while $\mathrm{Sr}^{2+}$ does not deviate from its formal ionic charge and no charge transfer is observed, the La charge changes from +3 to +2 for both adsorption modes (peroxo and superoxo), suggesting a covalent bond character between La and the charged molecule. In the present work no major charge transfer from the $\mathrm{Ca}$ ions is found: while the $\mathrm{Ca}$ ions are polarized, the Ca Bader charge stays +2 , similarly to $\mathrm{Sr}^{2+}$ on $\mathrm{SrTiO}_{3}(001),{ }^{23}$ as the charge is transferred from the subsurface $\mathrm{Ru}$ to the ionically bonded $\mathrm{O}_{2}{ }^{-}$at the surface. Preliminary DFT calculations (not shown) show a similar charge transfer mechanism on the related, SrO-terminated $\mathrm{Sr}_{3} \mathrm{Ru}_{2} \mathrm{O}_{7}(001)$ surface, suggesting that the difference is due to the energetic levels of the B-site ions.

Ultrathin oxide films that are only a few layers (L) thick are often considered as model systems for metal oxide surface chemistry. Calculations for ultrathin $\mathrm{MgO}$ films supported on a metal (Mo and $\mathrm{Ag}$ ) concluded that charge can be transferred from the metal support through the oxide film to the adsorbed oxygen. A comparison of $\mathrm{O}_{2}{ }^{-}$adsorbed on $\mathrm{MgO}(2 \mathrm{~L}) / \mathrm{Ag}(\Phi=3.1$ $\mathrm{eV})^{57}$ and $\mathrm{MgO}(3 \mathrm{~L}) / \mathrm{Mo}(\Phi=2.05 \mathrm{eV})^{57}$ showed that the adsorption energy ${ }^{\mathbf{1 9}}$ is smaller for the system with the higher work function. This suggests that the higher the work function the harder it is to transfer an electron to an adsorbate. The work function and $\mathrm{O}_{2}{ }^{-}$adsorption energy on $\mathrm{Ca}_{3} \mathrm{Ru}_{2} \mathrm{O}_{7}(001)$ of 2.7 and $0.72 \mathrm{eV}$, respectively, are similar to the values for the $\mathrm{MgO}(2 \mathrm{~L}) / \mathrm{Ag}$ system, 3.1 and $0.64 \mathrm{eV}$.

The formation of a full, adsorbed overlayer of $\mathrm{O}_{2}{ }^{-}$, and the availability of atomically-resolved nc-AFM allows to directly inspect the configurations of the adsorbate, thus providing a tight connection to the theoretical calculations. At low coverage, single $\mathrm{O}_{2}^{-}$could be imaged by AFM, but the application of a relatively low sample bias voltage $(\sim 100 \mathrm{mV})$ already resulted in interactions between the scanning tip and the molecules (Fig. 3 and 4). The interactions occurred for positive as well as negative sample bias voltages, and the exact threshold varied between 100 and $150 \mathrm{mV}$ depending on the tip-sample distance and the particular molecule. At higher sample bias voltages of roughly -1 and $+2 \mathrm{~V}$ the $\mathrm{O}_{2}{ }^{-}$is desorbed by the tip and the pristine surface is revealed (see Fig. $\mathrm{S} 2 \dagger$ ). The desorption at negative sample bias voltage, where the electrons tunnel from the molecule to the tip, might be facilitated by discharging the $\mathrm{O}_{2}{ }^{-}$as shown on anatase $\mathrm{TiO}_{2}(101) .{ }^{9}$

At increasing coverages the $\mathrm{O}_{2}^{-}$form an ordered $(2 \times 1)$ overlayer up to an apparent (in STM) saturation coverage of close to $1 \mathrm{ML}$. In contrast to isolated $\mathrm{O}_{2}{ }^{-}$the molecules in the overlayer can be imaged by STM without perturbation by the tip, except for the overlayer domain boundaries (Fig. 5b). Within the ordered overlayer the molecules are not moved about by the STM tip, probably because they are locked in by adjacent molecules, while at the boundaries tip-induced movement of molecules is possible. This agrees with the observation that after desorbing the $\mathrm{O}_{2}{ }^{-}$from a specific area by scanning at higher bias voltage, molecules from the surrounding area diffuse back to the area again during subsequent STM images taken at the usual, lower sample bias voltage (see Movie S1†).

Applications utilizing the ORR usually operate at much higher temperatures than the experimental temperatures of the present work. Therefore it is interesting how the interaction with $\mathrm{O}_{2}$ would change at realistic working conditions. As the tilting/rotation of the $\mathrm{RuO}_{6}$ octahedra strongly influences the 
adsorption energy of $\mathrm{O}_{2}{ }^{-}$in the different adsorption sites (B1/ B2 and B3/B4, see Fig. 2) it is appropriate to consider what happens to the $\mathrm{RuO}_{6}$ as the temperature is raised. The temperature dependence of the $\mathrm{Ca}_{3} \mathrm{Ru}_{2} \mathrm{O}_{7}$ structure was studied by Yoshida et $a l .{ }^{58}$ using neutron scattering up to $292 \mathrm{~K}$. Up to this temperature their data suggests that the $\mathrm{RuO}_{6}$ tilting angle slightly decreases but the structure does not undergo any dramatic changes. The orthorhombic structure of $\mathrm{Ca}_{2} \mathrm{RuO}_{4}$ and $\mathrm{Ca}_{3} \mathrm{Ru}_{2} \mathrm{O}_{7}$ is caused by $\mathrm{RuO}_{6}$ octahedral rotation and tilt. For $\mathrm{Ca}_{2} \mathrm{RuO}_{4}$, when both rotation and tilt disappear at elevated temperatures $(>357 \mathrm{~K})$, its structure becomes tetragonal. ${ }^{59}$ If $\mathrm{Ca}_{3} \mathrm{Ru}_{2} \mathrm{O}_{7}$ follows a similar trend (this has not been studied), adsorption of $\mathrm{O}_{2}$ as superoxo species would still be expected as it was calculated for tetragonal $\mathrm{Sr}_{3} \mathrm{Ru}_{2} \mathrm{O}_{7}$ (not published). However, due to the rather low adsorption energy of approximately $0.6 \mathrm{eV}$, the oxygen partial pressure would have to be substantially increased to achieve any $\mathrm{O}_{2}{ }^{-}$coverage at elevated temperatures: for example, if a simple Langmuir adsorption model is assumed, the $\mathrm{O}_{2}$ partial pressure necessary for a coverage of $0.5 \mathrm{ML}$ at $900 \mathrm{~K}$ is 1 bar. Regarding a possible change of the surface termination at elevated temperatures it can only be speculated that a $\mathrm{RuO}_{2}$-terminated surface would reconstruct. This is based on the fact that the $\mathrm{CaO}$ termination is by far the lowest-energy configuration, and that $\mathrm{SrTiO}_{3}$ (the only perovskite, where a significant number of surface studies are available) is known to form a variety of complex reconstructions with a structure that is sensitively dependent on the composition. ${ }^{60}$ However, in the present work no different termination than $\mathrm{CaO}$ was observed and studies of different perovskite-type materials after high temperature $\left(1000{ }^{\circ} \mathrm{C}\right)$ treatment under oxidizing conditions suggest that the surfaces are AO-terminated. ${ }^{61}$

\section{Conclusion}

The as-cleaved and unmodified $\mathrm{Ca}_{3} \mathrm{Ru}_{2} \mathrm{O}_{7}(001)$ surface activates molecular oxygen to $\mathrm{O}_{2}{ }^{-}$upon adsorption, with the charge stemming from the subsurface $\mathrm{RuO}_{2}$ layer. While the adsorption site and configurations are reflected well at the level of $\mathrm{vdW}$-DFT, more accurate theoretical approaches are necessary for a correct modelling of the charge redistribution and realistic binding energies. The advantages of a combined STM and ncAFM approach in case of adsorbates sensitive to electric field or tunnelling current are demonstrated; as single superoxo species are strongly influenced by the STM tip. When increasing the coverage, the $\mathrm{O}_{2}{ }^{-}$forms a full, $(2 \times 1)$ ordered monolayer that can be resolved by STM and AFM. The negative charge state of the molecules is evident in Kelvin probe and X-ray photoelectron spectroscopy. No dopants, defects, or low-coordinated sites are necessary to facilitate the activation of molecular $\mathrm{O}_{2}$ on this oxide surface.

\section{Author contributions}

D. H. performed the STM and XPS experiments and data analysis. D. H. and M. Se. performed the AFM measurements. W. M. S., F. M., and J. R. performed the DFT calculations. D. F., J. P., and Z. M. grew the sample. U. D. directed and supervised the project. D. H., W. M. S., F. M., M. Sc., and U. D. wrote the manuscript.

\section{Conflicts of interest}

The authors declare no competing financial interests.

\section{Acknowledgements}

This work was supported by the Austrian Science Fund (FWF project F45), the ERC Advanced Grant "OxideSurfaces", and the Vienna Scientific Cluster (VSC). Structural drawings were prepared using the program VESTA. ${ }^{62}$ The work at Tulane is supported by the U.S. Department of Energy under EPSCoR Grant No. DE-SC0012432 with additional support from the Louisiana Board of Regents.

\section{References}

$1 \mathrm{~J}$. A. Kilner and M. Burriel, Materials for intermediatetemperature solid-oxide fuel cells, Annu. Rev. Mater. Res., 2014, 44, 365-393.

2 M. Hayyan, M. A. Hashim and I. M. Alnashef, Superoxide ion: generation and chemical implications, Chem. Rev., 2016, 116, 3029-3085.

3 J. Rossmeisl, Z. W. Qu, H. Zhu, G. J. Kroes and J. K. Nørskov, Electrolysis of water on oxide surfaces, J. Electroanal. Chem., 2007, 607, 83-89.

4 G. M. Rupp, A. K. Opitz, A. Nenning, A. Limbeck and J. Fleig, Real-time impedance monitoring of oxygen reduction during surface modification of thin film cathodes, Nat. Mater., 2017, 16, 640-645.

5 R. Merkle and J. Maier, How is oxygen incorporated into oxides? A comprehensive kinetic study of a simple solidstate reaction with $\mathrm{SrTiO}_{3}$ as a model material, Angew. Chem., Int. Ed., 2008, 47, 3874-3894.

6 C. Schweitzer and R. Schmidt, Physical mechanisms of generation and deactivation of singlet oxygen., Chem. Rev., 2003, 103, 1685-1757.

7 M. Anpo, M. Che, B. Fubini and E. Garrone, Generation of superoxide ions at oxide surfaces, Top. Catal., 1999, 8, 189198.

8 M. Setvin, X. Hao, B. Daniel, J. Pavelec, Z. Novotny, G. S. Parkinson, M. Schmid, G. Kresse, C. Franchini and U. Diebold, Charge trapping at the step edges of $\mathrm{TiO}_{2}$ anatase (101), Angew. Chem., Int. Ed., 2014, 53, 4714-4716.

9 M. Setvin, J. Hulva, G. S. Parkinson, M. Schmid and U. Diebold, Electron transfer between anatase $\mathrm{TiO}_{2}$ and an $\mathrm{O}_{2}$ molecule directly observed by atomic force microscopy, Proc. Natl. Acad. Sci., 2017, 114, E2556-E2562.

10 Y. Zhao, B. T. Teng, X. D. Wen, Y. Zhao, Q. P. Chen, L. H. Zhao and M. F. Luo, Superoxide and peroxide species on $\mathrm{CeO}_{2}(111)$, and their oxidation roles, J. Phys. Chem. C, 2012, 116, 15986-15991.

11 M. Nolan, Healing of oxygen vacancies on reduced surfaces of gold-doped ceria, J. Chem. Phys., 2009, 130, 144702. 
12 Y. M. Choi, H. Abernathy, H. T. Chen, M. C. Lin and M. Liu, Characterization of $\mathrm{O}_{2}-\mathrm{CeO}_{2}$ interactions using in situ Raman spectroscopy and first-principle calculations, ChemPhysChem, 2006, 7, 1957-1963.

13 V. V. Pushkarev, V. I. Kovalchuk and J. L. D'Itri, Probing defect sites on the $\mathrm{CeO}_{2}$ surface with dioxygen, J. Phys. Chem. B, 2004, 108, 5341-5348.

14 X. Xia, R. J. Oldman and C. R. A. Catlow, Oxygen adsorption and dissociation on yttria stabilized zirconia surfaces, $J$. Mater. Chem., 2012, 22, 8594.

15 Y. Yan, M. M. Al-Jassim and S.-H. Wei, Oxygen-vacancy mediated adsorption and reactions of molecular oxygen on the $\mathrm{ZnO}(10 \overline{1} 0)$ surface, Phys. Rev. B: Condens. Matter Mater. Phys., 2005, 72, 161307.

16 D. Ma, Z. Wang, H. Cui, J. Zeng, C. He and Z. Lu, Firstprinciples study of $\mathrm{O}_{2}$ adsorption on Al-doped $\mathrm{ZnO}(10 \overline{1} 0)$ surface, Sens. Actuators, B, 2016, 224, 372-380.

17 A. Gurlo, Interplay between $\mathrm{O}_{2}$ and $\mathrm{SnO}_{2}$ : Oxygen ionosorption and spectroscopic evidence for adsorbed oxygen, ChemPhysChem, 2006, 7, 2041-2052.

18 Y. Cui, X. Shao, M. Baldofski, J. Sauer, N. Nilius and H. J. Freund, Adsorption, activation, and dissociation of oxygen on doped oxides, Angew. Chem., Int. Ed., 2013, 52, 11385-11387.

19 P. Frondelius, H. Häkkinen and K. Honkala, Adsorption and activation of $\mathrm{O}_{2}$ at $\mathrm{Au}$ chains on $\mathrm{MgO} / \mathrm{Mo}$ thin films., Phys. Chem. Chem. Phys., 2010, 12, 1483-1492.

$20 \mathrm{M}$. Che and A. J. Tench, Characterization and reactivity of molecular oxygen species on oxide surfaces, Adv. Catal., 1983, 32, 1-148.

21 A. Hellman, S. Klacar and H. Grönbeck, Low temperature CO oxidation over supported ultrathin MgO films, J. Am. Chem. Soc., 2009, 131, 16636-16637.

22 A. Gonchar, T. Risse, H. J. Freund, L. Giordano, C. Di Valentin and G. Pacchioni, Activation of oxygen on $\mathrm{MgO}$ : $\mathrm{O}_{2}{ }^{-}$radical ion formation on thin, metal-supported MgO(001) films, Angew. Chem., Int. Ed., 2011, 50, 2635-2638.

23 A. Staykov, H. Téllez, T. Akbay, J. Druce, T. Ishihara and J. Kilner, Oxygen activation and dissociation on transition metal free perovskite surfaces, Chem. Mater., 2015, 27, 8273-8281.

24 T. Akbay, A. Staykov, J. Druce, H. Téllez, T. Ishihara and J. A. Kilner, The interaction of molecular oxygen on $\mathrm{LaO}$ terminated surfaces of $\mathrm{La}_{2} \mathrm{NiO}_{4}$, J. Mater. Chem. A, 2016, 4, 13113-13124.

25 G. Kresse, From ultrasoft pseudopotentials to the projector augmented-wave method, Phys. Rev. B: Condens. Matter Mater. Phys., 1999, 59, 1758-1775.

26 J. Klimeš, D. R. Bowler and A. Michaelides, Van der Waals density functionals applied to solids, Phys. Rev. B: Condens. Matter Mater. Phys.B, 2011, 83, 195131.

27 J. Klimeš, D. R. Bowler and A. Michaelides, Chemical accuracy for the van der Waals density functional., J. Phys.: Condens. Matter, 2010, 22, 22201.

28 D. Halwidl, B. Stöger, W. Mayr-Schmölzer, J. Pavelec, D. Fobes, J. Peng, Z. Mao, G. S. Parkinson, M. Schmid, F. Mittendorfer, J. Redinger and U. Diebold, Adsorption of water at the SrO surface of ruthenates, Nat. Mater., 2015, 15, 450-455.

29 D. Halwidl, W. Mayr-Schmölzer, D. Fobes, J. Peng, Z. Mao, M. Schmid, F. Mittendorfer, J. Redinger and U. Diebold, Ordered hydroxyls on $\mathrm{Ca}_{3} \mathrm{Ru}_{2} \mathrm{O}_{7}(001)$, Nat. Commun., 2017, $8,23$.

30 A. V. Krukau, O. A. Vydrov, A. F. Izmaylov and G. E. Scuseria, Influence of the exchange screening parameter on the performance of screened hybrid functionals, J. Chem. Phys., 2006, 125, 224106.

31 M. Kaltak, J. Klimeš and G. Kresse, Cubic scaling algorithm for the random phase approximation: Self-interstitials and vacancies in Si, Phys. Rev. B: Condens. Matter Mater. Phys., 2014, 90, 54115.

32 M. Kaltak, J. Klimeš and G. Kresse, Low scaling algorithms for the random phase approximation: imaginary time and Laplace transformations, J. Chem. Theory Comput., 2014, 10, 2498-2507.

33 P. Liu, M. Kaltak, J. Klimeš and G. Kresse, Cubic scaling GW: towards fast quasiparticle calculations, Phys. Rev. B, 2016, 94, 165109.

34 F. J. Giessibl, Sensor for Noncontact Profiling of a Surface, US Pat. 2012/0131704 A1, 2012.

35 F. Huber and F. J. Giessibl, Low noise current preamplifier for qPlus sensor deflection signal detection in atomic force microscopy at room and low temperatures, Rev. Sci. Instrum., 2017, 88, 73702.

36 M. Setvín, J. Javorský, D. Turčinková, I. Matolínová, P. Sobotík, P. Kocán and I. Ošt'ádal, Ultrasharp tungsten tips - characterization and nondestructive cleaning, Ultramicroscopy, 2012, 113, 152-157.

37 J. I. J. Choi, W. Mayr-Schmölzer, F. Mittendorfer, J. Redinger, U. Diebold and M. Schmid, The growth of ultra-thin zirconia films on $\mathrm{Pd}_{3} \mathrm{Zr}(0001)$, J. Phys.: Condens. Matter, 2014, 26, 225003.

38 W. Bao, Z. Q. Mao, Z. Qu and J. W. Lynn, Spin valve effect and magnetoresistivity in single crystalline $\mathrm{Ca}_{3} \mathrm{Ru}_{2} \mathrm{O}_{7}$, Phys. Rev. Lett., 2008, 100, 247203.

39 G. Cao, K. Abboud, S. McCall, J. E. Crow and R. P. Guertin, Spin-charge coupling for dilute La-doped $\mathrm{Ca}_{3} \mathrm{Ru}_{2} \mathrm{O}_{7}$, Phys. Rev. B: Condens. Matter Mater. Phys., 2000, 62, 998-1003.

$40 \mathrm{~J}$. Tersoff and D. R. Hamann, Theory of the scanning tunneling microscope, Phys. Rev. B: Condens. Matter Mater. Phys., 1985, 31, 805-813.

41 A. Yurtsever, D. Fernández-Torre, C. González, P. Jelínek, P. Pou, Y. Sugimoto, M. Abe, R. Pérez and S. Morita, Understanding image contrast formation in $\mathrm{TiO}_{2}$ with force spectroscopy, Phys. Rev. B: Condens. Matter Mater. Phys., 2012, 85, 1-9.

42 R. Bechstein, Ć. Gonźalez, J. Schütte, P. Jeĺinek, R. Ṕerez and A. Kühnle, 'All-inclusive' imaging of the rutile $\mathrm{TiO}_{2}(110)$ surface using NC-AFM, Nanotechnology, 2009, 20, 505703.

$43 \mathrm{H}$. J. Lee and W. Ho, Single-bond formation and characterization with a scanning tunneling microscope, Science, 1999, 286, 1719-1722.

44 Y. Kim, K. Motobayashi, T. Frederiksen, H. Ueba and M. Kawai, Action spectroscopy for single-molecule 
reactions - Experiments and theory, Prog. Surf. Sci., 2015, 90, 85-143.

45 G. Cao, S. McCall, J. E. Crow and R. P. Guertin, Observation of a metallic antiferromagnetic phase and metal to nonmetal transition in $\mathrm{Ca}_{3} \mathrm{Ru}_{2} \mathrm{O}_{7}$, Phys. Rev. Lett., 1997, 78, 1751-1754.

46 J. Paier, M. Marsman and G. Kresse, Dielectric properties and excitons for extended systems from hybrid functionals, Phys. Rev. B: Condens. Matter Mater. Phys., 2008, 78, 1-4.

47 L. Schimka, J. Harl, A. Stroppa, A. Grüneis, M. Marsman, F. Mittendorfer and G. Kresse, Accurate surface and adsorption energies from many-body perturbation theory, Nat. Mater., 2010, 9, 741-744.

48 J. C. Rienstra-Kiracofe, G. S. Tschumper, H. F. Schaefer, S. Nandi and G. B. Ellison, Atomic and molecular electron affinities: photoelectron experiments and theoretical computations, Chem. Rev., 2002, 102, 231-282.

49 National Institute of Standard and Technology, NIST Computational Chemistry Comparison and Benchmark Database, NIST Standard Reference Database No. 101., 2006.

50 S. L. Dudarev, G. A. Botton, S. Y. Savrasov, C. J. Humphreys and A. P. Sutton, Electron-energy-loss spectra and the structural stability of nickel oxide: An LSDA+U study, Phys. Rev. B: Condens. Matter Mater. Phys., 1998, 57, 1505-1509.

51 S. Sadewasser and T. Glatzel, Kelvin Probe Force Microscopy, Springer Berlin Heidelberg, Berlin, Heidelberg, 2012.

52 P. O. Gartland, S. Berge and B. J. Slagsvold, Photoelectric work function of a copper single crystal for the (100), (110), (111), and (112) faces, Phys. Rev. Lett., 1972, 28, 738-739.

53 L. Gross, F. Mohn, P. Liljeroth, J. Repp, F. J. Giessibl and G. Meyer, Measuring the charge state of an adatom with noncontact atomic force microscopy., Science, 2009, 324, 1428-1431.

54 C. Puglia, A. Nilsson, B. Hernnäs, O. Karis, P. Bennich and N. Mårtensson, Physisorbed, chemisorbed and dissociated $\mathrm{O}_{2}$ on $\mathrm{Pt}(111)$ studied by different core level spectroscopy methods, Surf. Sci., 1995, 342, 119-133.
55 H. Li, J. I. J. Choi, W. Mayr-Schmölzer, C. Weilach, C. Rameshan, F. Mittendorfer, J. Redinger, M. Schmid and G. Rupprechter, Growth of an ultrathin zirconia film on $\mathrm{Pt}_{3} \mathrm{Zr}$ examined by high-resolution X-ray photoelectron spectroscopy, temperature-programmed desorption, scanning tunneling microscopy, and density functional theory, J. Phys. Chem. C, 2015, 119, 2462-2470.

56 M. M. Montemore, M. A. van Spronsen, R. J. Madix and C. M. Friend, $\mathrm{O}_{2}$ activation by metal surfaces: implications for bonding and reactivity on heterogeneous catalysts, Chem. Rev., 2017, DOI: 10.1021/acs.chemrev.7b00217.

57 L. Giordano, F. Cinquini and G. Pacchioni, Tuning the surface metal work function by deposition of ultrathin oxide films: Density functional calculations, Phys. Rev. B: Condens. Matter Mater. Phys., 2006, 73, 1-6.

58 Y. Yoshida, S.-I. Ikeda, H. Matsuhata, N. Shirakawa, C. Lee and S. Katano, Crystal and magnetic structure of $\mathrm{Ca}_{3} \mathrm{Ru}_{2} \mathrm{O}_{7}$, Phys. Rev. B, 2005, 72, 54412.

59 C. Alexander, G. Cao, V. Dobrosavljevic, S. McCall, J. Crow, E. Lochner and R. Guertin, Destruction of the Mott insulating ground state of $\mathrm{Ca}_{2} \mathrm{RuO}_{4}$ by a structural transition, Phys. Rev. B: Condens. Matter Mater. Phys., 1999, 60, R8422-R8425.

60 Z. Wang, A. Loon, A. Subramanian, S. Gerhold, E. McDermott, J. A. Enterkin, M. Hieckel, B. C. Russell, R. J. Green, A. Moewes, J. Guo, P. Blaha, M. R. Castell, U. Diebold and L. D. Marks, Transition from reconstruction toward thin film on the (110) surface of strontium titanate, Nano Lett., 2016, 16, 2407-2412.

61 J. Druce, H. Téllez, M. Burriel, M. D. Sharp, L. J. Fawcett, S. N. Cook, D. S. McPhail, T. Ishihara, H. H. Brongersma and J. A. Kilner, Surface termination and subsurface restructuring of perovskite-based solid oxide electrode materials, Energy Environ. Sci., 2014, 7, 3593-3599.

$62 \mathrm{~K}$. Momma and F. Izumi, VESTA 3 for three-dimensional visualization of crystal, volumetric and morphology data, $J$. Appl. Crystallogr., 2011, 44, 1272-1276. 